\title{
The effect of stenosis rate and Reynolds number on local flow characteristics and plaque formation around the atherosclerotic stenosis
}

\author{
XUEPING CHEN $^{1}$, YIYI ZHAN ${ }^{1,2}$, Yi FU ${ }^{3}$, JIANGGUO LIN ${ }^{1}$, YANRU JI ${ }^{1}$, \\ CHENGBI ZHAO ${ }^{4}$, YING FANG ${ }^{1}$, JIANHUA WU ${ }^{1} *$ \\ ${ }^{1}$ Institute of Biomechanics, School of Bioscience and Bioengineering, \\ South China University of Technology, Guangzhou, China. \\ ${ }^{2}$ Department of Electrical Engineering, School of Naval Architecture and Ocean Engineering, \\ Guangzhou Maritime University, Guangzhou, China. \\ ${ }^{3}$ Collaborative Innovation Center for Biomedicines and School of Medical Instruments, \\ Shanghai University of Medicine and Health Sciences, Shanghai, China. \\ ${ }^{4}$ Department of Naval Architecture and Ocean Engineering, School of Civil Engineering and Transportation, \\ South China University of Technology, Guangzhou, China.
}

\begin{abstract}
Purpose: Atherosclerosis causes plaque to build-up in arteries. Effect of the specific local hemodynamic environment around an atherosclerotic plaque on the thrombosis formation does not remain quite clear but is believed to be crucial. The aim of this study is to uncover the flow effects on plaques formation. Methods: To study the mechanically regulated plaque formation, the flow fields in artery blood vessels with different stenosis rates at various Reynolds numbers were simulated numerically with the two-dimensional axisymmetric models, and the hemodynamic characteristics around the plaque were scaled with stenosis rate and Reynolds number. Results: The results showed that increases of both Reynolds number and stenosis rate facilitated the occurrence of flow separation phenomenon, extended recirculation zone, and upregulated the maximum normalized wall shear stress near the plaque throat section while downregulated the minimal normalized wall shear stress at the front shoulder of plaque, as it should be; in the atherosclerotic plaque leeside of the recirculation zone, an obvious catch bond region of wall shear stress might exist especially under low Reynolds number with stenosis rate smaller than $30 \%$. This catch bond region in the plaque leeside might be responsible for the LBF (low blood flow)-enhanced formation of the atherosclerotic plaque. Conclusions: This work may provide a novel insight into understanding the biomechanical effects behind the formation and damage of atherosclerotic plaques and propose a new strategy for preventing atherosclerotic diseases.
\end{abstract}

Key words: stenosis rate, Reynolds number, atherosclerotic plaque, catch bond region, hemodynamics

\section{Introduction}

Atherosclerosis is a progressive disease characterized by localized plaques that form in the arterial wall. The stenosis on the arterial plaque leads to complex local hemodynamics environment, such as recirculation flow [6], [7], [9]. Severe stenosis may produce a large vortex region at downstream of the atherosclerotic plaque [5], which acts as a key hemodynamics factor for accumulation of atherosclerotic components, such as low-density lipoproteins to the plaque. Disturbed flow might damage the arterial intima at the vortex region, resulting in the deposition of platelet, blood lipids and other components at the injury site, and promote further growth of the atherosclerotic plaque [21]. The enlargement of plaque increases the stenosis rates of the artery, leading to hemodynamics-related pathological symptoms. For example, decreasing of coronary arteries diameter causes myocardial insuffi-

\footnotetext{
* Corresponding author: Jianhua Wu, Institute of Biomechanics, School of Bioscience and Bioengineering, South China University of Technology, Guangzhou, 510006, China. Phone: 020-39380608, e-mail: wujianhua@scut.edu.cn(J. Wu), yfang@scut.edu.cn (Y. Fang).

Received: August 28th, 2020

Accepted for publication: January 21st, 2021
} 
ciency and induced stable angina [24]. Furthermore, flow-induced coronary plaque rupture may lead to the formation of thrombosis and induce acute coronary syndromes, such as unstable angina, sudden cardiac death, and myocardial infarction. Therefore, research on the local flow characteristics around atherosclerotic plaque is of great significance to understand plaque formation.

Studies suggested that atherosclerotic plaques could significantly disturb the flow field around the stenosis place. The occurrence of complex flows in the arterial system had long been recognized from numerous studies. The study of Clark et al. showed that the flow behaves like a free jet for very small stroke volumes by using a flow visualization technique. Moreover, the extent of turbulence only depended on stenosis diameter and stroke volume while was independent of the distal downstream tube diameter [11]. Studies by Bit et al. showed that there is a positive correlation between relative disturbance and degree of stenosis in the latter crucial subcritical range of stenosis, and the degree of stenoses had a significant effect on the oscillatory shear index, relative residence time, and recirculation length [4], [5], [9], [13]. Banerjee et al. applied an axisymmetric cosine-shaped stenotic model to study the variation of blood flow characteristics near the stenosis in part of the coronary artery, and found that atherosclerotic plaque formation tended to start from very low flow Reynolds number [2]. Cassanova et al. measured the velocity and energy spectra in the distal region of modeled stenosis in a rigid tube, and found that significant flow disorder was created by a mild stenosis in pulsatile flow [8]. Furthermore, studies by Zhang et al. suggested that the reduction in diameter could resist the blood flows to the distal downstream of the stenosis site [30], suggesting that the plaques could lead to an insufficient blood replenishment of the downstream of vessels. All these studies indicated that the stenosis on the arterial plaque was the key factor for generating complex flow fields. The flow field in the human arterial system was not only affected by the geometric structure but also by $R e$. Moreover, clinical findings have shown that $R e$ in the human arterial ranged from less than 110 to over 5000 , where the flow status changed from laminar to turbulence [22].

A review by Sakamoto suggested that the characteristics of flow hemodynamics in the vessels are indeed affected by the geometric structure such as stenosis plaque and stents [21]. Several numerical and experimental analysis investigated the effect of local impact factors, such as scaffold influence and artery plaque development on the blood flow hemodynamics
[23], [24], [26], [29]. Yu et al. found that the 1D numerical model was able to capture the main features of the experimental pressure and flow waveforms with good reliability by comparing the flow characteristics of an experimental study of water-glycerin mixture flow in a 3D-printed silicone model of a complete circle of Willis with the simulated results by the 1D numerical model [29]. Although the 1D numerical model can capture the pressure and waveforms of blood flow, it is unable to obtain the distribution of wall shear stress along the vessel wall. Experimental and numerical flow analysis by Tomaszewski et al. showed that the areas of low wall shear stress values are located in close proximity to the stent struts [23], [24]. Tomaszewski et al. further found that compared to the normal diameter, stents with increased diameters have a higher risk of occurrence of the areas with low wall shear stress values. Moreover, excessive stent deformation can cause inflammation by injuring the vessel and can initiate the restenosis and thrombotic phenomena through the increased vessel diameter [24]. Wei et al., by both structural mechanical and hemodynamic analyses, found that stent structures indeed have impacts on the stress distribution within the plaque and artery as well as the local hemodynamic environment [26]. For instance, maximum stress mainly concentrated on the plaque and the maximum stress of plaque was observed in the contact sites near the end of the stent. Besides, the lower wall shear stress mainly located at sites around struts and links near the ends of the stent, while the higher wall shear stress mainly distributed in the middle regions near the center of the stent. Moreover, different shapes of stents have different impacts on the local hemodynamic environment.

Due to the technical limitations, the phenomenon of the blood flow was difficult to be monitored in vivo or accurately reproduced in experiment measurements in vitro. Computational fluid dynamics (CFD) was a valid and alternative approach used to precisely analyze the hemodynamics of vascular system [14]. In addition, CFD studies were more accurately compared with experiment measurements and advantages, such as repeatability of the data analysis, changeability of the model parameters, and evaluability of hemodynamic parameters in time and in space [14], were found. However, most previous numerical simulations had been focused on laminar flow with low $R e$ ranges [2], [8], [11], [30], and few studies gave a systematic study considering the effect of $R e$ in a wide range on flow patterns, and there was less knowledge of the specific local hemodynamic environment that in favor of cell adhesion to the atherosclerotic plaque. The mechano-regulation mechanism of the thrombosis 
formation was still unclear. For this reason, we hereby investigated the hemodynamic environment around atherosclerotic plaque by performing numerical simulation for the flow filed in artery blood vessels with different stenosis rates at various Reynolds number $R e$. In this work, the blood was assumed as a homogeneous and incompressible non-Newtonian fluid, the blood viscosity was estimated by Carreau model [10] and the stenosis vessels were modeled by the axisymmetric $2 \mathrm{D}$ models for simplicity. The main contributions of this work were: (1) first-time studied of the fraction of catch-bond regions for cell adhesion in the stenotic arteries; (2) systematically studied of the effects of different stenosis rates and Reynolds numbers ( $R e$ in a wide range from 110 to 4561) on the local flow characteristics; (3) found potential regions around the stenosis that may be easy to the formation of atherosclerotic vulnerable plaque; (4) the pressure drops developed by the stenosis can be an important parameter to characterize the severity of the atherosclerotic plaque.

\section{Materials and methods}

\section{Computational models}

In this study, the atherosclerotic plaque was approximated as a cosine shaped stenosis, similarly as in the previous work of Banerjee et al. [2], and the axisymmetric 2D models with three different stenosis rates were considered to investigate the effects of different severity of localized atherosclerotic plaques on flow characteristics. With a cylindrical coordinate system $O-x r$, in which $x$ and $r$ were the axial and radial coordinate with the coordinate system origin $(0,0)$ located at the inlet axis, respectively (Fig. 1A), the axisymmetric stenosis model was expressed as below,

$$
\frac{R_{(x)}}{R}= \begin{cases}1-S\left\{1-\cos \frac{x}{R} \pi\right\} / 2 & \text { for } x / R \in[8,10] \\ 1 & \text { for } x / R \notin[8,10]\end{cases}
$$

where $S(<1)$ denoted the stenosis rate of the vessel, $R$ and $R_{(x)}$ were the radii of the normal regions $(x<8 R$ and $x>10 R)$ and the $x$ places $(0 \leq x / R \leq 25)$, respectively. $R_{S}$ in Fig. $1 \mathrm{~A}$ was the radius at the narrowest place of the stenosis vessel. The axial length of the atherosclerotic plaque was set as $2 R(8 \leq x / R \leq 10)$ (Fig. 1A). For complicity, $R$ took unit scale for dimensionless. The mean radius of the left anterior descending (LAD) arteries $(R=1 \mathrm{~mm})$ was chosen as the unit size for the building of models, due to the high clinical incidence of the LAD arteries. To con- sider the moderate and severe stenosis in modeling, the stenosis rate $S$ was set to $0.30,0.50$ and 0.70 , respectively. To obtain a fully developed flow profile near the upstream of the stenosis and the outlet, we here let the upstream and downstream lengths of the normal regions of the vessel be $8 R$ and $15 R$, respectively (Fig. 1A). The present numerical analysis suggested that the soselected lengths on both sides of the atherosclerotic plaque were enough to reduce the influence of the distal boundary effect on the flow field near the stenosis.

\section{Governing equations}

The blood in this work was assumed as a homogeneous and incompressible non-Newtonian fluid, and its flow was governed by following incompressible Navier-Stokes equations,

$$
\begin{aligned}
\rho\left(\frac{\partial \mathbf{u}}{\partial t}+\mathbf{u} \cdot \nabla \mathbf{u}\right) & =-\nabla p+\nabla \cdot \boldsymbol{\tau}, \\
\nabla \cdot \mathbf{u} & =0,
\end{aligned}
$$

where $\mathbf{u}$ and $p$ represented respectively the fluid velocity vector and the pressure, $\frac{\partial \mathbf{u}}{\partial t}$ was equal to zero when using a steady numerical model, the two components of $\mathbf{u}$ in the axial and radial directions were denoted by $u$ and $v$, respectively, the blood density $(\rho)$ was a constant value $\left(1050 \mathrm{~kg} / \mathrm{m}^{3}\right)$, and $\tau$ expressed the stress tensor, which was formulated by

$$
\boldsymbol{\tau}=2 \eta(\dot{\gamma}) \mathbf{D},
$$

where $\mathbf{D}$ and $\dot{\gamma}$ were the deformation rate tensor and the shear rate, respectively, $\dot{\gamma}$ was related to the second invariant of $\mathbf{D}$, and $\eta$, a function of $\dot{\gamma}$, represented the blood viscosity. The blood has a non-Newtonian flow in which the blood viscosity depends upon the shear rate in the vessel. There were many non-Newtonian models that may be used for describing the viscosity of blood, such as Carreau model, Casson model, Cross model, Power law model, and Quemada model [9]. In this study, we chose the Carreau model to describe the material property of blood viscosity because the viscosity described by the Carreau model is the most accurate model to reflect the viscosity property of physiological blood in real compared to other viscosity models [20]. The expression of the Carreau model [10] was defined as below

$$
\eta(\dot{\gamma})=\eta_{\infty}+\left(\eta_{0}-\eta_{\infty}\right)\left[1+(\lambda \dot{\gamma})^{2}\right]^{\frac{n-1}{2}}
$$

where $\eta_{\infty}=3.45 \times 10^{-3} \mathrm{~kg} /(\mathrm{m} \cdot \mathrm{s}), \eta_{0}=5.6 \times 10^{-2} \mathrm{~kg} /(\mathrm{m} \cdot \mathrm{s})$, $n=0.3568$ and $\lambda=3.313 \mathrm{~s}[10]$. 
(A)

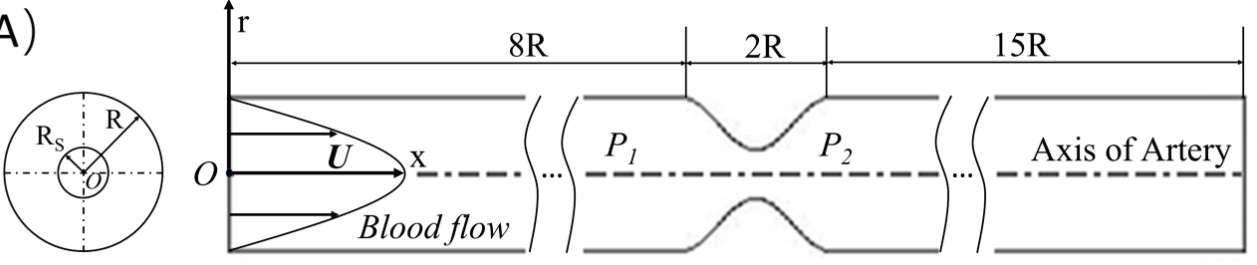

(B)

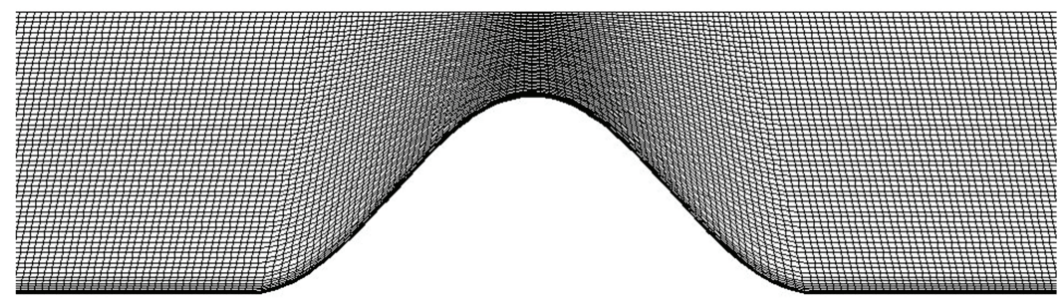

(C) $\quad \mathrm{L}_{\mathrm{r}}+\mathrm{L}_{\mathrm{B}}$

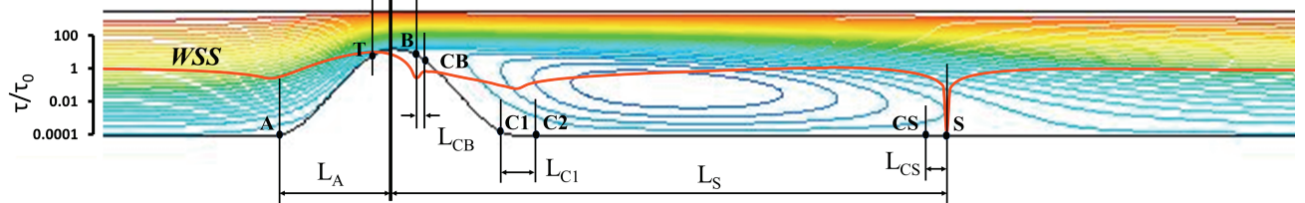

Fig. 1. The computational model building and characteristic parameters defining. (A) with a cylindrical coordinate system $O-\mathrm{xr}$, in which $x$ and $r$, respectively were the axial and radial coordinate with the coordinate system origin $(0,0)$ located at the inlet axis.

$R$ was the radius, the total length of the stenosed vessel was $25 R$. (B) The typical mesh of the computational model for the atherosclerotic plaque domain. (C) The definitions of the target points and characteristic parameters.

The points $A, T, B$, and $S$ were the 1st valley point, maximum, 2nd valley point, and stagnation, respectively.

The points $C B, C 1, C 2, C \mathrm{~S}$ were the borders between the catch bond region and slip bond region.

The lengths of $L_{A}, L_{T}, L_{B}$, and $L_{\mathrm{S}}$ were the axial distances from stenosis center to the 1st valley point, maximum,

2nd valley point, and stagnation, respectively. The lengths of $L_{C B}, L_{C 1}, L_{C S}$ were the axial distances of point pair $B$ and $C B, C 1$ and $C 2$, and $C S$ and $S$, respectively

\section{Boundary conditions $(B C)$}

At the inlet $(x=0)$, a steady non-Newtonian flow was considered for all simulations and a fully developed parabolic velocity profile was imposed by

$$
\begin{gathered}
u=2 U\left[1-\left(\frac{r}{R}\right)^{2}\right], \quad 0 \leq r \leq R, \\
v=0,
\end{gathered}
$$

where $U$ was the average of the axial velocity $u$ over cross section of the inlet, and $r$ was the radial distance away from $x$-axis of the vessel (Fig. 1A). A rigid and no-slip boundary condition was implemented at the vessel wall and the plaque, and the stress-free boundary condition was applied at the outlet.

The resistance coefficient $(K)$ of the plaque was defined by

$$
K=\frac{\left(P_{1}-P_{2}\right)_{\text {Stenosis }}-\left(P_{1}-P_{2}\right)_{\text {Health }}}{\frac{1}{2} \rho U^{2}},
$$

where, $P_{1}$ and $P_{2}$ were the pressures at the inlet and the outlet of the vessel (Fig. 1A), respectively

\section{Numerical scheme}

Imposed with the boundary conditions mentioned above for the inlet, the outlet and the wall of the vessel, the governing equations of blood flow were solved by ANSYS FLUENT 14.0 (ANSYS Inc., USA) with use of the finite volume method. The Reynolds number $R e$ was set to $110,154,217$, $304,435,598,839,1177,1561,2320,3251$ and 4561 , respectively, because $R e$ was shown to be ranged from less than 100 to over 5000 for the blood flow in the human arterial system [22]. The steady simulation method was considered here. The laminar model was used for $R e<2320$, while the k- $\varepsilon$ turbulence model was used for $R e \geq 2320$. The SIMPLE algorithm was utilized for the pressure-velocity coupling. A coupled solver was performed with a second-order upwind scheme for the momentum spatial discretization. The convergence criterion was set to be $10^{-9}$ for both continuity and velocity residuals. 
Computational models were meshed as rectangular grids by using the ANSYS ICEM 14.0 software (ANSYS, Inc., USA). The mesh for all the computational domains (including upstream, downstream and the stenosis domain) were refined with 7 layers at the near-wall, and the height ratio was set to 1.2. A typical mesh of the computational model for the atherosclerotic plaque domain was shown in Fig. 1B. The mesh independence was supposed to be achieved when the difference of the wall shear stress, as well as the pressure between two successive simulations was less than $1 \%$. One sample case was simulated for each stenosis rate as a representation. If the mesh independence was reached, the model was applied to all the other Reynolds numbers with the same stenosis rate.

\section{The definition of characteristic parameters}

A typical streamline pattern of flow was shown in Fig. $1 \mathrm{C}$, the red line in it was the corresponding pattern of wall shear stress on the vessel with an atherosclerotic plaque, where $\tau$ and $\tau_{0}$ were respectively the wall shear stresses of the vessel with or without the stenosis fraction. Based on the distribution of wall shear stress, we introduced eight points on the arterial wall and plaque ( $A, T, B, C B, C 1, C 2, C S$ and $S$ ). The points $A, T, B$, and $S$ were the 1 st valley point, maximum, 2nd valley point, and stagnation, respectively. The points $C B, C 1, C 2, C S$ (from the 2 nd valley point to stagnation) were the borders between the catch bond region and slip bond region. The lengths of $L_{A}, L_{T}, L_{B}$, and $L_{S}$ were the axial distances from stenosis center to the 1 st valley point, maximum, 2nd valley point, and stagnation, respectively. The lengths of $L_{C B}, L_{C 1}, L_{C S}$ were the axial distances of point pair $B$ and $C B, C 1$ and $C 2$, and $C S$ and $S$, respectively.

\section{Results}

\section{Streamline patterns of blood flow around the atherosclerotic plaque}

To examine effects of the Reynolds number $R e$ and the stenosis rate $S$ on the local hemodynamics environment, we numerically simulated the flow field in the arterial blood vessel with three different stenosis rates $(30 \%, 50 \%$ and $70 \%)$ at various values of Reynolds number $(110,154,217,304,435,598,839,1177$, 1561, 2320, 3251 and 4561) (Chapter 2). The fluid motion should be dominated by laminar flow for $R e$ below the critical Reynolds number $(=2320)$ or by turbulence flow for $R e$ over the critical Reynolds number. Variation of streamline pattern of flow around an atherosclerotic plaque versus $S$ and $R e$ was shown in Fig. 2, in which the typical streamline patterns from laminar to turbulence flow were plotted for each stenosis rate with five Reynolds numbers from $154,304,598,2320$ to 4561 . Various recirculation zones generated through the vortex flow were obvious shown in the streamline patterns for laminar and turbulence flows with different stenosis (Fig. 2). The recirculation zone was believed to be responsible for formation of atherosclerosis [6]. A physiological reason was that the blood flow stagnated and refluxed locally in the recirculation zone, as a result, various proteins or adhesive molecules, platelets, leukocytes and so on were allowed to form the mesh at the inner wall of the arteries, triggering the early events in the atherosclerotic plaque formation.

We observed from Fig. 2 that the flow separation phenomenon occurred at the plaque throat section (leeward of the narrowest position of the vessel), and the downstream separated-flow reattached at the distal location of the posterior plaque; the distance from the

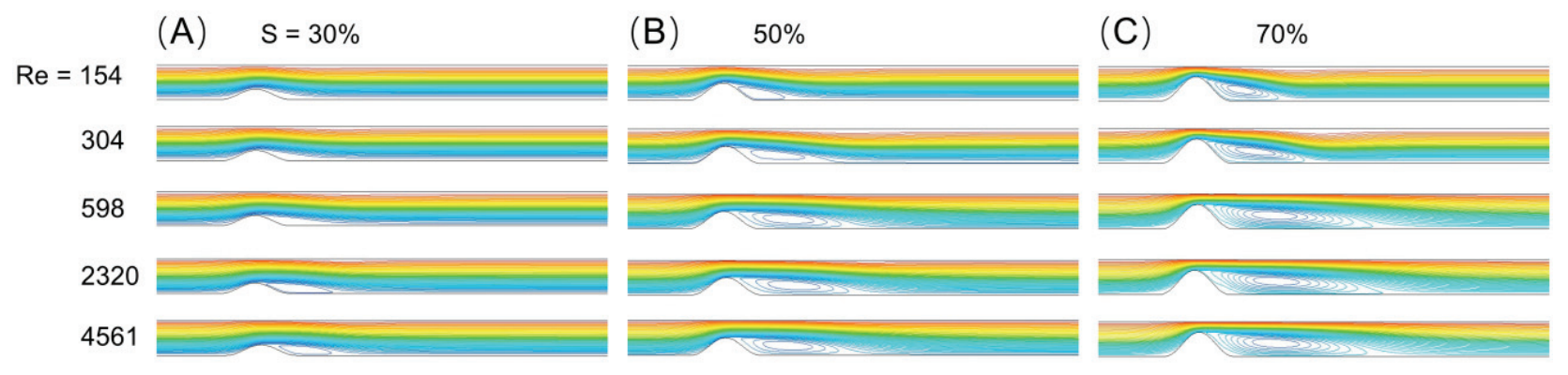

Fig. 2. Typical streamline patterns of blood flow. Figs. 2A, B and C showed the streamlines of five typical Re for three different stenosis rates (A: 30\%, B: 50\% and C: 70\%). Re =154, 304, 598 represented the laminar flow, $R e=2320$ was the critical Reynolds number, $R e=4561$ represented turbulence flow; Stenosis rates, from moderate to severe, were set to $30 \%, 50 \%$, and $70 \%$. The length of the recirculation zone increased with both $R e$ and stenosis rate 
separated- to reattached-site of the vessel increased significantly with the stenosis rate for a given Reynolds number, so did the recirculation zone area in the flow field, showing a stenosis rate-enhanced flow separation phenomenon for flows over atherosclerotic plaque; and for both moderate and severe stenosis, increasing Reynolds number made the recirculation zone area extended in laminar flow at $R e<600$ but almost steady in turbulent flow and laminar flow at $R e \geq 600$, so did the distance from the separated- to reattached-site of the vessel. The stenosis rate-enhanced flow separation for flows over atherosclerotic plaque were also observed in previous work at low Reynolds number [2], and reflected to the effect of Reynolds number and stenosis rate on the flow separation-mediated vortex generation on the downstream of the plaque. This phenomenon was more significant under severe stenosis.

\section{The distributions of wall shear stress around the atherosclerotic plaque}

The distributions of the dimensionless wall shear stress $\tau / \tau_{0}$ for different stenosis rates and various Reynolds numbers were plotted in Fig. 3, which showed a multiphasic pattern of wall shear stress on stenosis site. The $\tau / \tau_{0}$ pattern was composed of four stages in series. Along with the axial direction, $\tau / \tau_{0}$, at the 1 st stage, was decreasing until it reached its 1 st valley point $\left(x=x_{A}\right)$ at front shoulder of the stenosis, and at the 2 nd stage, increased steeply to its maximum at the point $\left(x=x_{T}\right)$ near center of the stenosis site firstly and then dropped quickly to its 2 nd valley point $\left(x=x_{B}\right)$ or the flow separation point on the rear shoulder of the stenosis, further at the 3rd stage, undergone a pathway, which was a parabolic type for $30 \%$ stenosis with $R e \leq 304$ (Fig. 3D and E) or "M" types for others (Fig. 3) without regard to the slightly pulsing occurred at high Reynolds number $R e \geq 2320$ (Figs. 3G, H), until it reached its stagnation point $\left(x=x_{\mathrm{S}}\right)$; lastly, at the 4th stage, $\tau / \tau_{0}$ would increase up to one, which meant that the disturbed-flow was fully developed again (Fig. 3). The high gradient of wall shear stress on the plaque site came from that the blood flow was accelerated and decelerated at the front and rear plaque respectively, leading to a mechanical damage risk of the plaque throat section, on which the wall shear stress was very high in comparison with that on normal vessel wall and the low level of wall shear stress at the front and rear stenosis shoulders might be required for adhesion and recruitment of cell to the damage plaque site.

\section{Fluid-mechanics based characteristics around the atherosclerotic plaque}

To further scale the effects of Reynolds number and stenosis rate on the feature locations around the atherosclerotic plaque, we here introduced four relative lengths (fluid mechanics based characteristics), such as $L_{T}\left(=\left|x_{T} / R-9\right|\right), L_{S}\left(=\left|x_{S} / R-9\right|\right), L_{A}\left(=\left|x_{A} / R-9\right|\right)$ and $L_{B}\left(=\left|x_{\mathrm{B}} / R-9\right|\right)$, being the relative axial distances from the stenosis center or throat section to the maxi-
(A)

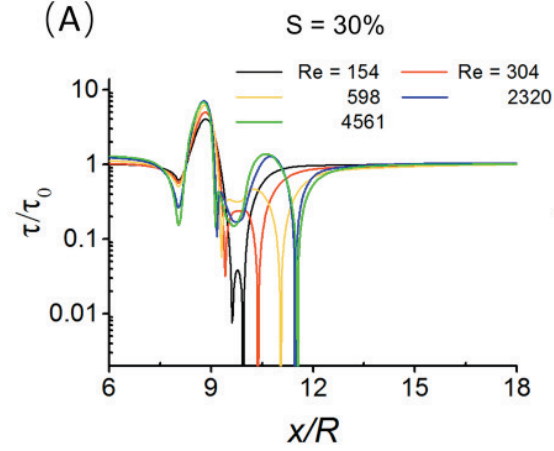

(B)

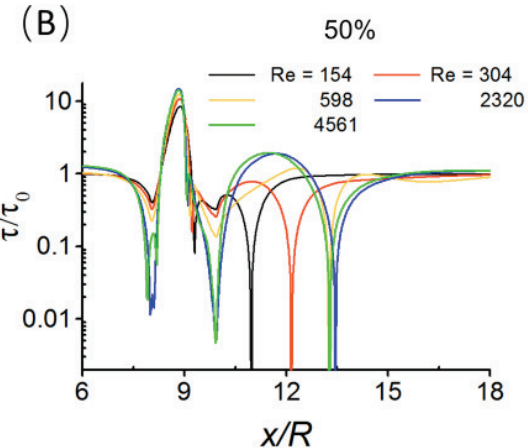

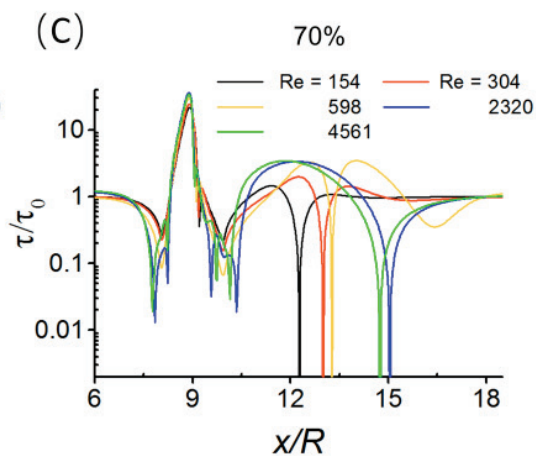

(D) $\operatorname{Re}=154$
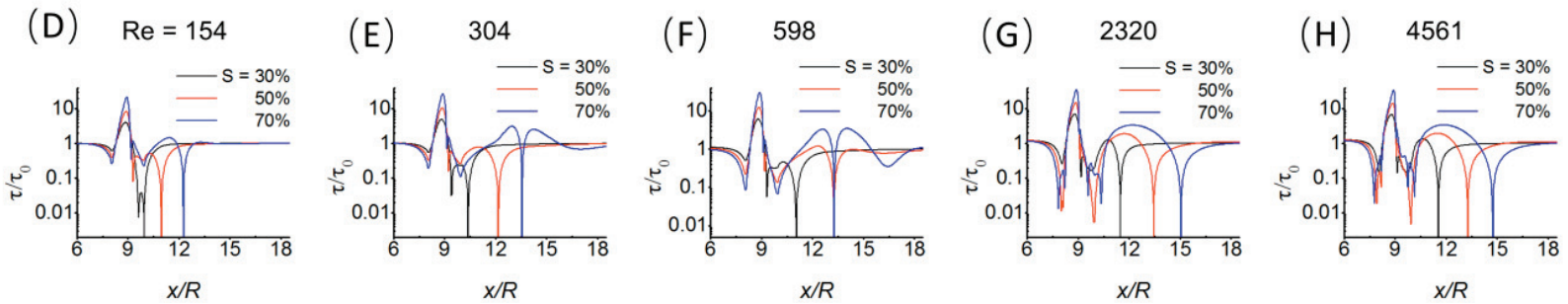

Fig. 3. Typical patterns of wall shear stress. FiguresA-H show the distribution of the dimensionless wall shear stresses $\left(\tau / \tau_{0}\right)$ for different stenosis rates and various $R e$. The wall shear stress were significantly disturbed by the atherosclerotic plaque, especially for larger $R e$ and higher stenosis rate 
mum, the stagnation, the 1 st and the 2 nd valley points, at which the wall shear stress took its maximum $\tau_{\max }$, zero, $\tau_{A}$ and $\tau_{B}$, respectively, in the pattern of the wall shear stress, respectively (Fig. 1C). It followed from Fig. 4A and B that $L_{T}$ increased steeply as Re increased from 110 to 4561 for each stenosis rate, so did $\tau_{\max } / \tau_{0}$, meaning that the location of $\tau_{\max }$ at the atherosclerotic plaque would move away (to windward) from the stenosis center with increasing $R e$; and $L_{T}$ decreased by at least $30 \%$ while $\tau_{\max } / \tau_{0}$ doubled as $S$ increased either from $30 \%$ to 50 or from $50 \%$ to $70 \%$, saying that increasing stenosis rate made the location of $\tau_{\max }$ at the plaque appeared more closer to the stenosis center.

The 1 st and 2 nd valley points respectively located on the front and rear of the plaque might be responsible for promoting the plaque damage in the complex flow, because that the weak mechano-stability of newly vulnerable formed plaque [19] might be even weaker enhanced by the low wall shear stress at the two valley points. Plots of $L_{A}$ and $L_{B}$ against $R e$ with three different stenosis rates showed (Figs. 4C, D) that, each of $L_{\mathrm{A}}$ and $L_{\mathrm{B}}$ decreased with either $R e$ or $S$, indicating that the high Reynolds number stream in more severe stenosis made both the 1st and the 2nd valley points close more to the stenosis center in comparison with low Reynolds number stream in lower stenosis. The results also showed that $\tau_{A} / \tau_{0}$ decreased with either $R e$ or $S$, while $\tau_{B} / \tau_{0}$ increased with either $R e$ or $S$ (Figs. 4 E, F). The maximum of the normal fluid stress on the plaque occurred at the
1 st valley point shared with the minimal wall shear stress $\tau_{A}$ on the front shoulder of the plaque. As $R e$ increased from 110 to $4561, L_{A}$ declined from 0.87 to 0.79 , from 0.83 to 0.75 and from 0.81 to 0.71 , for $30 \%$, $50 \%$ and $70 \%$ stenosis rates respectively (Fig. 4C), meaning that the maximal normal stress was located at the shoulders of the plaques for lower Reynolds number flow.

The stagnation regions were believed to be deeply involved in the formation and propagation of atherosclerotic plaques. Low wall shear stress, being induced by the flow vortex within the recirculation zone, might enhance both mitosis and apoptosis of endothelium cell near the stagnation site [17]. So the leaky junction induced by the high cell turnover rate might occur at neighborhood of the stagnation site, to which the accumulation of low-density lipoproteins was allowed, while a significant low-density lipoprotein accumulation in the arterial wall was the early event in the initiation and progression of atherosclerotic diseases [17]. But, the coregulation of Reynolds number and stenosis rate on the stagnation position was not fully clear. Here, we observed that, as a scale of the recirculation zone or vortex length, $L_{S}$ increase steeply with $R e$ first and then reached to a plateau as $R e$ was over 1177 for each stenosis, and obviously increased with the stenosis rate for each Reynolds number (Fig. 4G), demonstrating that increasing both stenosis rate and Reynolds number $(\leq 1177)$ would make the stagnation point away from the plaque (Fig. 2).
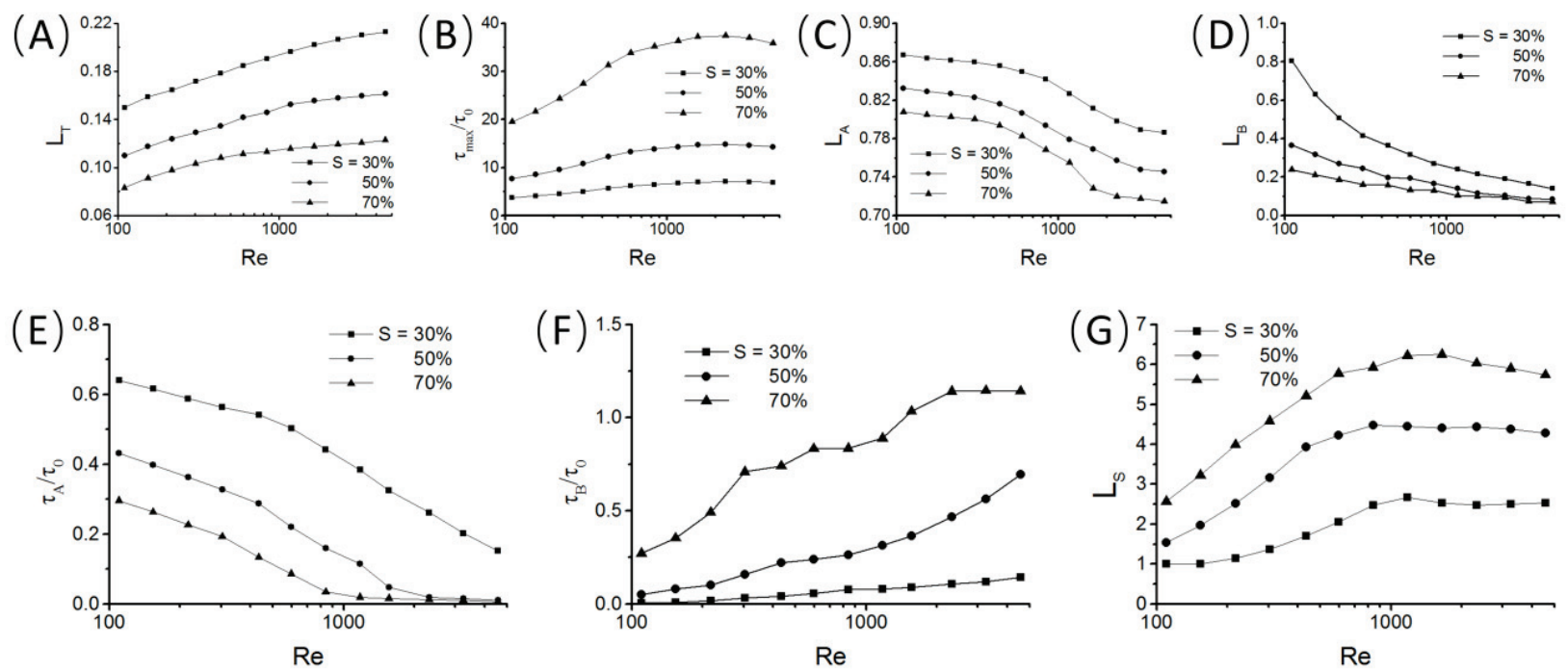

Fig. 4. The effect of $R e$ and stenosis rate on the change of characteristic parameters and dimensionless wall shear stress: $\mathrm{A}$ - the $L_{T}$ increased with $R e$ while decreased with stenosis rate, B - the $\tau_{\max } / \tau_{0}$ increased with either $R e$ or stenosis rate, $\mathrm{C}$ - the $L_{A}$ decreased with either $R e$ or stenosis rate, $\mathrm{D}$ - the $L_{B}$ decreased with either $R e$ or stenosis rate, $\mathrm{E}$ - the $\tau_{A} / \tau_{0}$ decreased with either $R e$ or stenosis rate, $\mathrm{F}-$ the $\tau_{B} / \tau_{0}$ increased with either $R e$ or stenosis rate, $\mathrm{G}-$ the $L_{\mathrm{S}}$ increased with either $R e$ or stenosis rate 


\section{The resistance coefficient $K$}

of the atherosclerotic plaque

The atherosclerotic plaque of arteries would resist blood flows to the posterior root of stenosis, leading to an insufficient blood replenishment of the downstream of vessels [30]. The resistance coefficient $(K)$ of the plaque was referred to the loss of the pressure drop induced by the stenosis relative to the health vessel, and estimated by Eq. (8) (Chapter 2). A typical pattern of pressure on the vessel wall with $50 \%$ stenosis and $R e$ of 110 was plotted in Fig. 5A, which demonstrated that the atherosclerotic plaque induces the loss of pressure drop in the vessel. All other patterns of pressure were shown in Fig. S1 of the Appendix materials at the end of this paper. The results showed a similar trend of the pressure drop for all the cases (Fig. S1). The effects of $R e$ and $S$ on the loss of pressure drop were reflected to the resistance coefficient $K$ (Fig. 5B). It was found that $K$ decreased with $R e$ obviously for severe $70 \%$ stenosis and slightly for moderate $50 \%$ stenosis, remained almost constant for $30 \%$ stenosis, but increased obviously with the stenosis rate $S$ for any given $\operatorname{Re}$ (Longitudinal comparison) (Fig. 5B). These results suggested that the resistance coefficient had a positive correlation to the stenosis level but a negative correlation to the Reynolds number, and the effect of stenosis level on the resistance coefficient was more profound than that of Reynolds number. Atherosclerotic patients usually had a breath shortness, such as hypoxia symptoms, even when they were doing a minor exercise. The shortness might come from an increased vascular resistance that influenced the blood perfusion in the distal organ. The present results illustrated that the many fold increase of the resistance was induced by stenosis rather than Reynolds number.

\section{The fraction of catch-bond regions \\ for cell adhesion in the recirculation zone}

Flowing platelet adhesion was essential for hemostasis on the injured vessel site [15], [28], and it was believed to flow-enhanced process through a catch-slip bond transition mechanism [18], which said that increasing hemodynamic forces would make the lifetime of adhesive molecule bond prolonged first and then shortened [28]. The force threshold was about $16 \mathrm{dyn} / \mathrm{cm}^{2}$ for flowing platelet adhesion, and 25-30 dyn $/ \mathrm{cm}^{2}$ for adhesion of neutrophils and HL60 [15]. So, the mechanical microenvironment might induce vessel damage near neighborhood of stagnation site [17], but be propitious to adhering of platelets and leukocytes to the injured site, coming from requirements of platelet hemostasis and leukocyte inflammation reaction. It suggests that the mechanical microenvironment near the stagnation site is in favor of cell adhesion or the catch bond formation of adhesive molecules. To test it, we examined whether or not there existed a catch bond region (CBR) (Fig. 6), in which the wall shear stress at the vessel site was slower than the wall shear stress threshold and enhanced adhesion of cell on vessel, for various Reynolds number and three different stenosis. The catch bond region CBR in recirculation zone on the stenosis leeside between the point $B$ and $S$ were measured with the wall shear stress threshold of $16 \mathrm{dyn} / \mathrm{cm}^{2}$ for the vWF-induced platelet adhesion and or $35 \mathrm{dyn} / \mathrm{cm}^{2}$ for E-selectioninduced HL-60 cell roll adhesion, and $\beta$, the CBR fraction of the recirculation zone, were calculated by $\beta=\left(L_{C S}+L_{C B}+L_{C 1}\right) / L_{S B}$ (Fig. 1C), where $L_{S B}\left(=x_{S}-x_{B}\right.$ $\geq 0$ ) were the axial distances from the stagnation to the 2 nd valley points, moreover, $L_{C S}\left(=x_{\mathrm{S}}-x_{\mathrm{CS}} \geq 0\right)$, $L_{C B}\left(=x_{C B}-x_{B} \geq 0\right)$ and $L_{C 1}\left(=x_{C 2}-x_{C 1} \geq 0\right)$ were the
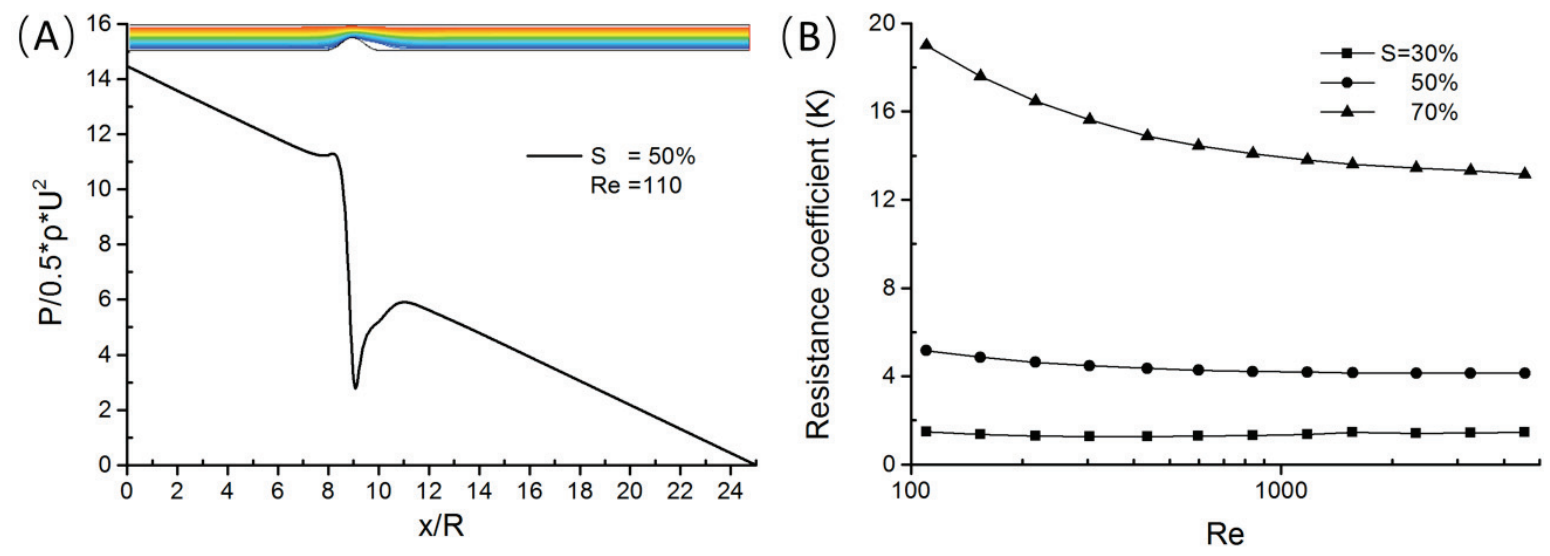

Fig. 5. The resistance coefficient $(K)$ of the atherosclerotic plaque changed with $R e$ and stenosis rates:

A - a representative result of the effect of atherosclerotic plaque on the distribution of pressure along the vessel wall, the atherosclerotic plaque can significantly increase the loss of pressure drop in the vessel. The stenosis rate is $50 \%$ and $R e$ is 110 , $\mathrm{B}$ - the resistance coefficient decreased with $R e$ while increased with stenosis rate 

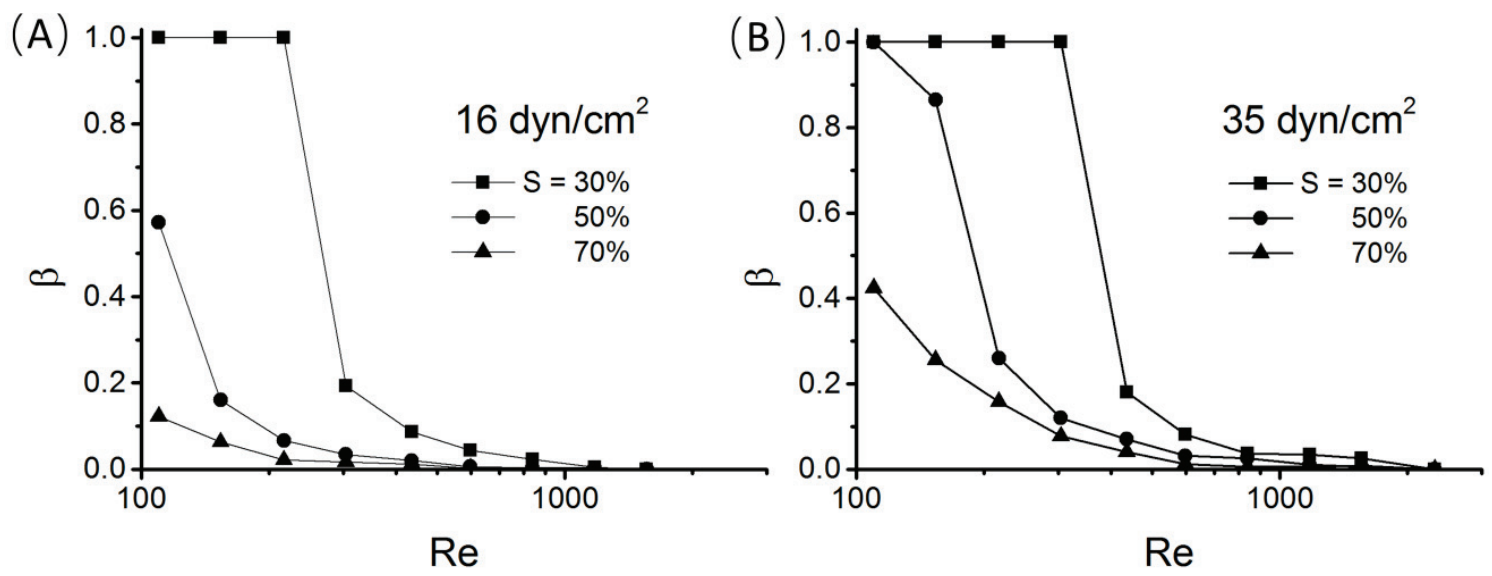

Fig. 6. The effect of $R e$ and stenosis rate on the change of catch bond region (CBR) fraction. Figures $6 \mathrm{~A}$ and $\mathrm{B}$ showed the catch bond region (CBR) fraction decreased with either Re or stenosis rate for both platelets and white cells

lengths of three sub-CBRs, being near stagnation point, the separation point and the one between other two, respectively, and the wall shear stress on vessel took its threshold at positions for $x=x_{C S}, x_{C B}, x_{C 1}$ and $x_{C 2}$ $\left(x_{S} \geq x_{C S} \geq x_{C 2} \geq x_{C 1} \geq x_{C B} \geq x_{B}\right)$ and was below the threshold if $x \in\left\{\left(x_{S}-x_{C S}\right) \cup\left(x_{C B}-x_{B}\right) \cup\left(x_{C 2}-x_{C 1}\right)\right\}$ (Fig. 1C).

Plots of $\beta$ against $R e$ for various stenosis rates (Figs. 6A, B) showed that, at $30 \%$ stenosis rate, $\beta$ first remained $100 \%$ high level at lower $R e$ smaller than either 217 for the force threshold of $16 \mathrm{dyn} / \mathrm{cm}^{2}$ or 304 for the force threshold of $35 \mathrm{dyn} / \mathrm{cm}^{2}$ first, and then dropped from these high levels to a low level of $20 \%$ about for all the force thresholds; and at $50-70 \%$ stenosis, the plateau of $100 \%$ high level in $\beta$ pattern diminished, and $\beta$ was decreased quickly with both $R e$ and $S$. This demonstrated that at low Reynolds number flow especially with lower stenosis of $30 \%$ about, the atherosclerotic plaque leeside would exist an obvious catch bond region, which might cover all the leeside in the recirculation zone, enhancing the adhesion of flowing platelets and leucocytes. It was believed that the formation of atherosclerotic plaque tended to start from very low blood flow (LBF) [2]. This suggested that the catch bond mechanism of adhesive molecules might be responsible for the LBFenhanced formation of the atherosclerotic plaque formation.

\section{Discussion}

A detailed knowledge on the flow pattern at atherosclerotic plaque was essential for estimating the risk of disease. Reynolds number and geometric structure were two key factors that impact the distribution of flow patterns in the vascular [6], [7], [9], [13]. At low Reynolds numbers, flows tend to be dominated by laminar flow, while at high Reynolds numbers $(>2320)$, flows tend to be dominated by turbulence flow. As atherosclerotic plaque was more likely to form under low Reynolds number condition, therefore, many researchers tend to focus on the study of the blood flow field under low Reynolds number. However, clinical findings have shown that $R e$ in the human arterial ranges from less than 110 to over 5000 [22]. Study by Cassanova et al. [8] found that significant flow disorder was created by a moderate stenosis with $R e$ about 318. Moreover, arteries with severe stenosis under high Reynolds numbers can also generate very low wall shear stress in the local sites such as the stagnation point. Therefore, fully considering the effects of different stenosis rates and Reynolds number on the local flow pattern of the atherosclerotic plaque was significant. Because the flow-mechanical regulation involved in the plaque formation was not fully understood, to uncover this phenomenon, in this study, the effect of stenosis rates and Re on the hemodynamics factors around atherosclerotic plaque were systematically investigated.

Plenty of clinical reports and studies showed that stenosis plaque of arteries does not have particular shapes [16]. Moreover, the shapes of the stenosis plaque could be varied with the locations and the degree of stenosis in the real arteries. In order to simplify the specific problems, several studies considered stenosis plaque in the patient-specific arteries (such as coronary artery) as elliptical [16] or a cosine shape [2]. In this study, the stenosis model was developed by referring to the previous work of Banerjee et al. in which the plaque approximated as cosine shape stenosis [2]. 
In a specific geometry model, the distribution of hemodynamics in the stenotic arteries can be affected by the properties of blood, such as viscosity. The blood has a non-Newtonian flow in nature, in which the blood viscosity depends upon the shear rates in the vessel. Study by Bit et al. [9] suggested that different non-Newtonian rheological models, such as the Carreau model, Casson model, Cross model, Power law model, and Quemada model, lead to different distributions of the flow field in the stenotic vessel. According to the measurement experiment by Pratumwal et al. [20], compared to other non-Newtonian rheological models, the viscosity described by the Carreau model is the most accurate to reflect the viscosity property of whole blood in real. Therefore, in this study, we chose the Carreau model to describe the blood viscosity.

Atherosclerosis was a disease that led to plaque formation at the specific sites of the arterial trees through intimal inflammation, necrosis, fibrosis, and calcification. In the selectin-induced recruitment of flowing leukocytes to vascular injury sites for the formation of plaques, the flow-enhanced cell adhesion was believed to be mediated through the interaction of PSGL-1 with selectin. This interaction was governed by a "catch-slip bond transition" mechanism, which was a flow force-based cell adhesion mechanism [18]. Previous work showed that leukocyte activation was essential for innate immunity and resistance to pathogen infection and injury, and platelet activation was essential for hemostasis on the injured organ or tissue [28]. Studies showed that catch bond behavior only existed under the low wall shear stress condition and beyond the required optimal value (threshold value) will lead to a slip bond behavior [28]. Yago et al. found that when the hemodynamic force was lower than threshold, increasing hemodynamic forces can strengthen the binding force between the adhesion cells. However, when the hemodynamic forces was higher than the threshold value, increasing hemodynamic forces can weaken the binding rate between the adhesion cells [28]. Our results showed that the wall shear stress at the stagnation point was zero. It suggested that the mechanical microenvironment near the stagnation site was in favor of cell adhesion or the catch bond formation of adhesive molecules. Moreover, our results showed that the catch bond region fraction $\beta$ in the atherosclerotic plaque leeside varied with the $R e$ and stenosis rates (Fig. 6). When $R e$ was higher than 1561, all the hemodynamic forces on the arterial wall was over the threshold value (for both platelets and leukocytes) and the catch-bond regions disappear on the vessel wall. In addition, when stenosis rate was up to $70 \%$, the critical $R e$ for the catch- -bond regions disappear was even as low as 598 (Fig. 6). These results revealed that lower stenosis rates and smaller $R e$ could promote the cell adherent reaction at the downstream regions of the atherosclerotic plaque.

The recirculation zone generated by the vortex flow was considered to be an important flow phenomenon for the further formation of atherosclerosis. The physiological significance of the recirculation zone was that the blood flow stagnated and refluxed locally in this area, which allowed platelets and other adhesion cells and molecules to form the mesh at the inner wall of the arteries. This may cause even more severe stenosis rate of the plaque. Apart from this, the stagnation point in the recirculation zone was also significant for the formation and propagation of atherosclerosis, as the wall shear stress in the stagnation point is very low. Studies showed that the rate of mitosis or apoptosis of cells can be significantly increased by the low wall shear stress on the endothelium [17]. Moreover, leaky junction induced by the high cell turnover rate easily occurs near the stagnation point, allowing low-density lipoprotein accumulating into the arterial wall. Researchers proposed that the initiation and progression of the atherosclerotic disease involves a significant accumulation of lowdensity lipoproteins in the arterial wall [17]. This suggested that the stagnation region may be an important area for the formation and propagation of atherosclerosis. As novel-formed plaques usually do not have good mechanical stability and it was susceptible to disturbances by the complex flow [19], therefore, this may be one reason why the rupture of the atherosclerotic vulnerable plaques easily happened at the recirculation zone (downstream of the stenosis site) [3]. Hence, the present study can give the prediction of the possible site for the formation of atherosclerotic vulnerable plaque (Fig. 4G).

Studies suggested that the high wall shear stress might strengthen the mechanical properties of the atherosclerotic plaques [12]. Our results indicated that the mechanical stability at the center of atherosclerotic plaque can be greatly strengthened by the severe stenosis (Fig. 4A). Moreover, the present results also suggested that $\tau_{\max } / \tau_{0}$ increased obviously with either $R e$ or stenosis rate (Fig. 4B). These results explained why arterial plaque with severe stenosis was more stable than that with moderate stenosis [3], and further suggested that vulnerable plaques could prone to appear in lower Reynolds number. Unlike the wall shear stress, the high normal fluid stress on the plaques might make the plaque more vulnerable [12]. Moreover, the results in Fig. 4C showed that the maximal normal stress prone to located at the root of the plaques. This 
might partially explain why the plaque damage appeared frequency in the root of the plaque, and was consistent with the clinical data that most plaques rupture at the fibrous cap edge of the plaques [25].

It is well known that severe stenosis of arteries may lead to a fatal situation. Accurate diagnosis and measurement of the presence of stenosis in arteries is very important for the patients to avoid facing fatal consequences. Study by Kamangaret et al. [16] suggested that the pressure across the artery could drop significantly with the presence of stenosis in the vessel. The present study showed that the pressure suddenly drops when blood flow encounters stenosis and then starts to recover after having passed through the stenotic area. Moreover, a higher stenosis rate has larger pressure drops from the inlet to the outlet (Fig. S1). On the other hand, the loss of pressure drop was reflected to the resistance coefficient $K$. It was found that severe stenosis had a larger $K$ (Fig. 5B). Therefore, the pressure drops developed due to the presence of stenosis is a very important parameter to understand the severity of the blockage, which may help to build an alternative tool to diagnosis the stenosis in arteries.

Many numerical and experimental studies regarding the blood flow hemodynamics in the stenotic vessels with the effect of implanted objects such as stents were conducted [23], [24], [26]. Studies by Tomaszewski et al. suggested that the results of the distribution of averaged velocity vectors measured by applying the particle image velocimetry (PIV) method were very close to the CFD approach. This suggested that numerical methods can well represent the experimental tests to study hemodynamic characteristics. Moreover, using numerical methods allows not only for analyzing the flow characteristics, but also indicating the regions of low and oscillating shear stresses, which may impede upon results of coronary angioplasty [23], [24]. Furthermore, compared with experimental measurements, numerical methods were more accurate and have many advantages like repeatability of the data analysis, changeability of the model parameters, and evaluability of hemodynamic parameters in time and in space.

Although many excellent results such as detailed values for the wall shear stress, oscillatory shear index, and relative residence time can be easily obtained by numerical simulation, basic challenges such as the wall zone effect, mesh density and mesh sensitivity studies are need to be solved to make the numerical results reliable [1], [23], [24], [27]. Studies suggested that to fully reflect the wall zone effect on the hydrodynamic characteristics occurring close to the vessel walls, special mesh, such as structured meshes like a prism, needs to be constructed on the parietal layers, which can help to accurately capture the flow characteristics near the arterial wall [1], [23]. Tomaszewski et al. suggested that the $\mathrm{Y}+$ parameter could help to determine the size of the first mesh, and the width of the boundary layer, the $\mathrm{Y}+$ value for the closed channels should be below 1 [23]. Moreover, the mesh sensitivity study is essential for determining the number of mesh and to ensure if the mesh-independent results were obtained for each simulation. The artificially denser mesh in certain regions of the vessels, such as the plaque area with a stenotic channel, could make the results very sensitive to numerical aspects of influential and doubtful. Therefore, a proper mesh density is essential to obtain reliable results. In our present study, the difference of WSS/pressure between two successive simulations for each case was controlled within less than $1 \%$ with the use of quadrilateral mesh. This suggested that the numerical results were not sensitive to the mesh size. Hence, we considered that the generated mesh in this study is good enough for running the current simulations.

Although interesting results were obtained from the present study, there are still some limitations that need to be pointed out. The first limitation is that we only use the simple 2D axisymmetric models which are quite different from the real artery geometry. The reason is that the main scientific problem we want to solve in this study is the effect of stenosis rate and Reynolds number on hemodynamics near the plaque. Using a real artery geometry will bring many other uncontrolled factors in the model such as curves and tapers, which could influence the simulation results and make the problem even complex. Moreover, applying the 2D model can help us to reduce computing resources and save time. Another limitation is that only the steady numerical model was used for the numerical simulations. Moreover, the current simulations were not compared with experimental tests. Lastly, we only used the Carreau non-Newtonian rheological model to mimic the material property of blood viscosity. In our future work, we will fully consider these aspects of limitations and take as much real artery geometry as possible into the researches to make our studies more robust.

\section{Conclusions}

In summary, to uncover the phenomenon of flowmechanical regulation involved in the plaque formation, in this paper, the effect of stenosis rates and Reynolds number on the blood flows around atherosclerotic plaque were investigated with an axisymmetric 
2D model of the stenosis vessel. The present study novelly analyzed the ranges of the locations and fraction of catch-bond regions for cell adhesion around the atherosclerotic plaque and further studied the change of resistance coefficient with different stenosis rate and Reynolds number. The results illustrated that increases of both Reynolds number and stenosis facilitated occurrence of flow separation phenomenon, extended recirculation zone, and upregulated the maximum normalized wall shear stress near the plaque throat section while downregulated the minimal normalized wall shear stress at the front shoulder of plaque; and an obvious catch bond region might exist obviously in the atherosclerotic plaque leeside of in the recirculation zone, especially under low Reynolds number with low stenosis rate small $30 \%$, being responsible for the LBF-enhanced formation of the atherosclerotic plaque formation. This study may present a new insight in understanding the biomechanical effects on the formation and damage of atherosclerotic plaques. Moreover, the pressure drops developed by the stenosis could be a very important parameter to understand the severity of the blockage which may help to build an alternative tool to diagnosis the stenosis rate in arteries.

\section{Data availability}

The datasets used to support the findings of this study are included within the article.

\section{Conflicts of interest}

All authors declare that they have no conflict of interest involved in this manuscript.

\section{Acknowledgements}

We thank the National Natural Science Foundation of China and the Fundamental Research Funds for the Central Universities supporting this work. This study was funded by National Natural Science Foundation of China 11432006 (Jianhua Wu), 11672109 (Ying Fang), 31771012 (Jiangguo Lin) and 31500759 (Jiangguo Lin), the Fundamental Research Funds for the Central Universities, SCUT, D2156960 (Jianhua Wu), and the Guangzhou Science Technology Program 201707010062 (Jiangguo Lin).

\section{References}

[1] BAHRAmiAn A., Simultaneous effects of mesh refinement, grid configuration and wall boundary condition on prediction of pressure gradients and velocity profiles of microparticles in a conical fluidized bed, Particuology, 2019, 43, 123-136.
[2] Banerjee M.K., Ganguly R., Datta A., Variation of Wall Shear Stress and Flow Characteristics Across Cosine Shaped Stenotic Model with Flow Reynolds Number and Degree of Stenosis, International Journal of Fluid Mechanics Research, 2010, 37 (6), 530-552.

[3] Bentzon J.F., Otsuka F., Virmani R., Falk E., Mechanisms of plaque formation and rupture, Circ. Res., 2014, 114 (12), $1852-1866$.

[4] Bit A., Alblawi A., Chattopadhyay H., Quais Q.A., BEnIM A.C., RAHIMI-GorJI M., Do H.T., Three dimensional numerical analysis of hemodynamic of stenosed artery considering realistic outlet boundary conditions, Comput. Methods Programs Biomed., 2020, 185, 105163.

[5] Bit A., Chattopadhay H., Acute Aneurysm is more Critical than Acute Stenoses in Blood Vessels: a Numerical Investigation Using Stress Markers, BioNanoScience, 2018, 8 (1), 329-336.

[6] Bit A., ChattopadhyAy H., Numerical investigations of pulsatile flow in stenosed artery, Acta Bioeng. Biomech., 2014, 16 (4), 33-44.

[7] Bit A., Ghagare D., Rizvanov A.A., Chattopadhyay H., Assessment of Influences of Stenoses in Right Carotid Artery on Left Carotid Artery Using Wall Stress Marker, Biomed. Res. Int., 2017, 2935195.

[8] Cassanova R.A., Giddens D.P., Disorder distal to modeled stenoses in steady and pulsatile flow, Journal of Biomechanics, 1978, 11 (10), 441-453.

[9] Chattopadhyay H., Himadri, Bit A., Arindam , Assessment of rheological models for prediction of transport phenomena in stenosed artery, Progress in computational fluid dynamics: An international journal, 2014.

[10] ChO Y.I., Kensey K.R., Effects of the non-Newtonian viscosity of blood on flows in a diseased arterial vessel. Part 1. Steady flows, Biorheology, 1991, 28 (3-4), 241-262.

[11] CLARK C., The propagation of turbulence produced by a stenosis, Journal of Biomechanics, 1980, 13 (7), 591-604.

[12] Coppola G., CARo C., Arterial geometry, flow pattern, wall shear and mass transport: potential physiological significance, J. R. Soc. Interface, 2009, 6 (35), 519-528.

[13] Eswari J.S., Majdoubi J., Naik S., Gupta S., Bit A., RAHIMI-GoRJI M., SALEEM A., Prediction of stenosis behaviour in artery by neural network and multiple linear regressions, Biomech. Model Mechanobiol., 2020, 19 (5), 1697-1711.

[14] Gramigna V., Caruso M.V., Rossi M., Serraino G.F., RENZULli A., Fragomeni G., A numerical analysis of the aortic blood flow pattern during pulsed cardiopulmonary bypass, Comput. Methods Biomech. Biomed. Engin., 2015, 18 (14), 1574-1581.

[15] Huang B., Ling Y., Lin J., FAng Y., Wu J., Mechanical regulation of calcium signaling of HL-60 on P-selectin under flow, Biomed. Eng. Online, 2016, 15 (Suppl. 2), 153.

[16] Kamangar S., Badruddin I.A., AhAmad N.A., Govindaraju K., Nik-Ghazali N., Salman Ahmed N.J., BADARUdin A., Yunus KHAN T.M., The Influence of Geometrical Shapes of Stenosis on the Blood Flow in Stenosed Artery, Sains Malaysiana, 2017, 46 (10), 1923-1933.

[17] KIM S., GiDDENS D.P., Mass transport of low density lipoprotein in reconstructed hemodynamic environments of human carotid arteries: the role of volume and solute flux through the endothelium, J. Biomech. Eng., 2015, 137 (4), 041007-0410017.

[18] Marshall B.T., Long M., PiPer J.W., Yago T., MceVer R.P., ZHU C., Direct observation of catch bonds involving celladhesion molecules, Nature, 2003, 423 (6936), 190-193. 
[19] Meng H., Tutino V.M., Xiang J., SiddiQui A., High WSS or low WSS? Complex interactions of hemodynamics with intracranial aneurysm initiation, growth, and rupture: toward a unifying hypothesis, AJNR. American Journal of Neuroradiology, 2014, 35 (7), 1254-1262.

[20] Pratumwal Y., Limtrakarn W., Muengtaweepongsa S., PhaKdeESAN P., InTHARAKHAM K., Whole blood viscosity modeling using power law, Casson, and Carreau Yasuda models integrated with image scanning U-tube viscometer technique, Songklanakarin Journal of Ence and Technology, 2017, 39 (5), 625-631.

[21] SAKamoto A., Jinnouchi H., Torit S., Virmani R., FinN A.V., Understanding the Impact of Stent and Scaffold Material and Strut Design on Coronary Artery Thrombosis from the Basic and Clinical Points of View, Bioengineering (Basel), 2018, 5 (3).

[22] Stein P.D., SABBah H.N., Turbulent blood flow in the ascending aorta of humans with normal and diseased aortic valves, Circ. Res., 1976, 39 (1), 58-65.

[23] Tomaszewski M., Sybilski K., Baranowski P., MAŁACHOWSKI J., Experimental and numerical flow analysis through arteries with stent using particle image velocimetry and computational fluid dynamics method, Biocybernetics and Biomedical Engineering, 2020, 40 (2), 740-751.

[24] TOMASZEWSKi M., SyBILSKi K., MAŁACHOWSKi J., WolAŃSKI W., BUSZMAN P.P., Numerical and experimental analysis of balloon angioplasty impact on flow hemodynamics improvement, Acta of Bioengineering and Biomechanics, 2020, 22 (3).

[25] Van Der Wal A.C., BecKer A.E., Atherosclerotic plaque rupture - pathologic basis of plaque stability and instability, Cardiovascular Research, 1999, 41 (2), 334-344.

[26] Wei L., Leo H.L., Chen Q., Li Z., Structural and Hemodynamic Analyses of Different Stent Structures in Curved and Stenotic Coronary Artery, Front Bioeng. Biotechnol., 2019, 7, 366.

[27] Woodward P., Colella P., The numerical simulation of two-dimensional fluid flow with strong shocks, Journal of Computational Physics, 1984, 54 (1), 115-173.

[28] Yago T., Lou J., Wu T., Yang J., Miner J.J., Coburn L., López J.A., CruZ M.A., DONG J.-F., MCINTIRE L.V., MCEVER R.P., ZHU C., Platelet glycoprotein Ib $\alpha$ forms catch bonds with human WT $v W F$ but not with type $2 B$ von Willebrand disease $v W F$, Journal of Clinical Investigation, 2008, 118 (9), 3195-3207.

[29] Yu H., Huang G.P., Ludwig B.R., Yang Z., An In-Vitro Flow Study Using an Artificial Circle of Willis Model for Validation of an Existing One-Dimensional Numerical Model, Ann. Biomed. Eng., 2019, 47 (4), 1023-1037.
[30] Zhang J.-M., Zhong L., LuO T., HuO Y., TAN S.Y., Wong A.S.L., Su B., Wan M., Zhao X., Kassab G.S., LeE H.P., Khoo B.C., Kang C.-W., BA T., TAN R.S., Numerical simulation and clinical implications of stenosis in coronary blood flow, BioMed Research International, 2014, 514729-514729.

\section{Appendix}

\section{The patterns of pressure drop along the arterial wall}

In Figure S1, the patterns of pressure drop along the arterial wall for different Reynolds number and stenosis rate is shown. The results showed that the trend of pressure drop for all the cases is similar. Moreover, the atherosclerotic plaque may induce the loss of pressure drop in the vessel. The results further showed that a higher stenosis rate has larger pressure drops from the inlet to the outlet (Fig. S1). This suggested that the pressure drops developed due to the presence of stenosis is a very important parameter to understand the severity of the blockage.

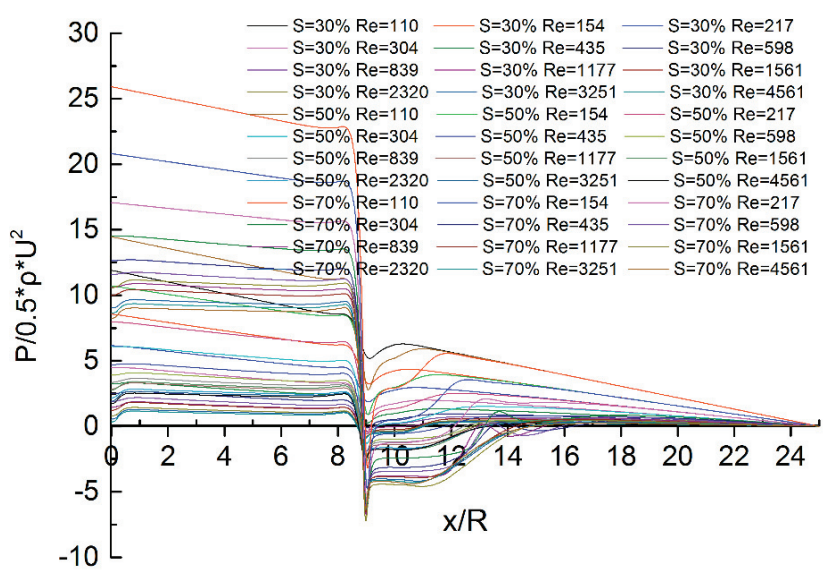

Fig. S1. Patterns of pressure drop along the arterial wall 